\title{
Reconstruction of Chronic Peroneal Tendon Tears
}

\author{
${ }^{1}$ Christopher E Gross, ${ }^{2}$ Beau J Kildow, ${ }^{3}$ Selene G Parekh
}

\begin{abstract}
Peroneal tendon tears are one of many causes of chronic lateral ankle pain. The goal of surgery is to restore function and provide dynamic stabilization. Surgical repair is indicated for tears comprising of less than $50 \%$ of the cross-sectional area of the tendon. If the peroneal tear is greater than $50 \%$ and the tissue bed remains mobile, an allograft reconstruction may be performed. In this case report, reconstruction using a peroneal tendon allograft was performed on a 21-year-old female with chronic lateral ankle pain and weakness after retearing her peroneal brevis 10 weeks status postprimary repair. Technique involved excising the portion of diseased tendon and anastomosing the proximal and distal ends to a peroneal tendon allograft. Six weeks postoperation, patient regained full range of motion and strength with minimal pain.
\end{abstract}

Keywords: Peroneal, Allograft, Reconstruction, Chronic lateral ankle pain.

How to cite this article: Gross CE, Kildow BJ, Parekh SG. Reconstruction of Chronic Peroneal Tendon Tears. J Foot Ankle Surg (Asia-Pacific) 2014;1(2):60-64.

\section{Source of support: Nil}

Conflict of interest: None

\section{INTRODUCTION}

Pathology of the peroneal tendons is one of the many causes of chronic lateral ankle pain, though it is often underdiagnosed. ${ }^{1,2}$ While isolated tears of the peroneus brevis and longus are rare, fissuring and longitudinal splitting of the brevis and longus tendons have been reported as a cause of chronic ankle pain and functional instability. Delays in diagnosis are common as up to $40 \%$ of peroneal tendon disorders are missed at first evaluation. ${ }^{3}$ When recognized early, direct repair is possible with good results. ${ }^{4-6}$

The peroneal tendons are typically injured in an inversion ankle sprain or in chronically unstable ankles. However, the pathogenesis of chronic peroneal tendon ruptures is not entirely clear. Many theories have been suggested, including a zone of critical hypovascularity, 7,8 mechanical impingement from the fibular groove, ${ }^{9-11}$

\footnotetext{
${ }^{1}$ Fellow, ${ }^{2}$ Resident, ${ }^{3}$ Physician

1,3 Department of Orthopedic and Foot and Ankle, Duke University Durham, NC, USA

${ }^{2}$ Department of Orthopedic, Duke University, Durham, NC, USA
}

Corresponding Author: Beau J Kildow, Resident, Department of Orthopedic, 4709 Creekstone Dr Durham, NC 27703, USA Phone: 402-215-3651, e-mail: beau.kildow@duke.edu incompetence of the peroneal retinaculum, ${ }^{12,13}$ the presence of a sharp posterior fibular ridge, ${ }^{9-11}$ dynamic compression between the peroneus longus and brevis tendons, ${ }^{14}$ hypertrophy of the peroneal tubercle ${ }^{15}$ or the presence of a peroneus quartus muscle. ${ }^{16,17}$ The most common location for longitudinal peroneus brevis tendon tears is at the fibular groove, ${ }^{8}$ while the most common location for a peroneus longus tear is at the peroneal tubercle, at the entry of the cuboid tunnel. At the fibula, both the peroneus longus and brevis have reduced vascularity. ${ }^{7}$

Patients can present with advanced pathology of on or both tendons, such that neither can be salvaged in their entirety. Many have a history of at least one failed surgical procedure to attempt a primary repair or anastomosis of the peroneus brevis and longus tendons.

The goal of surgery is to provide dynamic stabilization of the ankle and restore the function of the peroneal tendons. Once both tendons have had an extensive synovectomy and debridement, a treatment decision is made. In tendons that have greater than $50 \%$ of their cross-sectional areas debrided due to degeneration, the stump is tenodesed to the other peroneal tendon (assuming it is viable). If both tendons have greater than $50 \%$ degeneration, an algorithm detailed by Redfern and Myerson is utilized. ${ }^{18}$ If the proximal muscle bellies are not mobile, then a tendon transfer is performed (FHL). If there is some proximal muscle excursion and if the tissue bed is scarred, than a staged silicone rod reconstruction is performed. If the tissue bed is mobile than an allograft reconstruction is performed (Flow Chart 1).

We present a case report of a patient with chronic lateral ankle pain who underwent a peroneal allograft procedure for chronic peroneal tendinopathy.

\section{CASE REPORT}

A 21-year-old female presented to the office with a chief complaint of left lateral ankle pain after twisting her ankle dismounting a horse 2 weeks prior. Her pain improved with the CAM boot, RICE therapy, and NSAIDS. The patient described the pain as achy, sharp, and constant with pain score at $5 / 10$. She also reported clicking and stiffness on the lateral side of her ankle. On physical exam, the patient was tender over the anterior talofibular ligament (ATFL) ligament and peroneal tendons. She had full symmetric range of motion (ROM) about the foot and ankle but experienced pain with active and passive 
subtalar eversion. There was no apparent ligamentous laxity or crepitation. Peroneal muscle strength was $4 / 5$. Magnetic resonance imaging (MRI) revealed longitudinal tears of peroneal brevis and longus (Fig. 1).

After failing nonoperative management, she underwent a procedure involving peroneal tendon repair with fibular groove deepening. She was doing well until, after completing 10 weeks of PT, the patient returned to clinic after reinjuring her ankle while twisting her ankle on the foot stirrup while riding her horse.

On physical exam, patient was again tender over the ATFL and peroneal tendons. She had moderate ecchymosis on the posterolateral ankle over her surgical scar. She also had a positive anterior drawer sign in plantar flexion. She continued to be symptomatic 6 weeks after reinjuring her ankle so an MRI was obtained. It revealed an acute tear in the previously repaired peroneal brevis tendon (Fig. 2). We elected to return the patient to the operating room. Intraoperatively, both peroneal ten-

Flow Chart 1: Surgical techniques recommended for extent of peroneal pathology
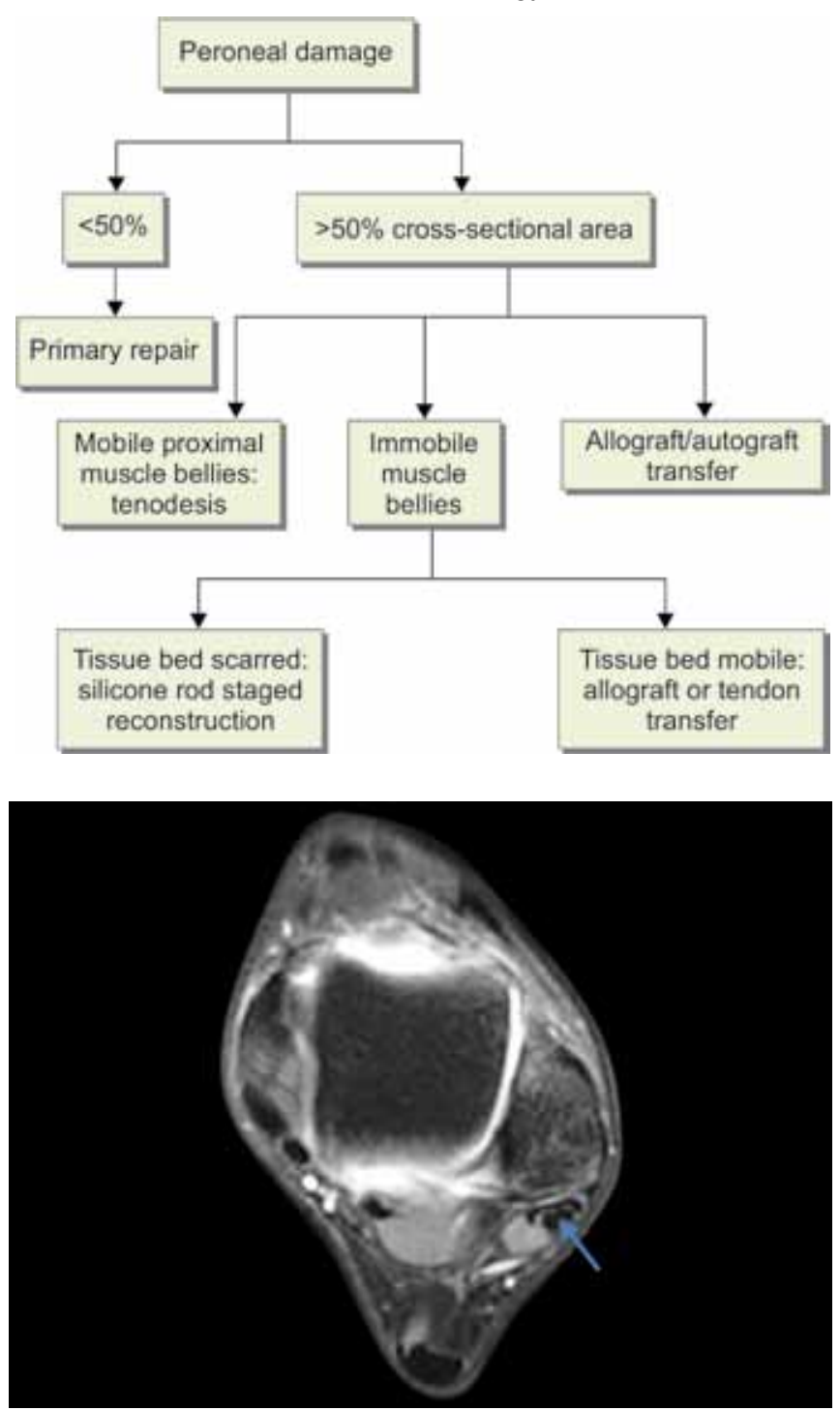

Fig. 1: T2 MRI revealing peroneal brevis and longus tears (arrow) dons were found to have profound degeneration. The patient underwent peroneal brevis allograft procedure as described below. At 16 weeks postoperative, she was able to ambulate without pain with $4+/ 5$ peroneal strength.

She was participating fully in activity until she reinjured her ankle again about 1 year out from allograft reconstruction. She twisted her ankle while horseback riding, causing persistent ankle weakness, stiffness and $6 / 10$ pain. Her physical exam was identical to when she first injured her ankle with $4 / 5$ peroneal muscle strength. She completed 3 weeks of PT without any improvement. An MRI was ordered which revealed tears in peroneus longus and brevis tendons. The patient elected to proceed with third procedure involving peroneal tendon debridement $v s$ repair $v s$ allograft reconstruction. During the operation, no tear was appreciated in the native peroneus longus or brevis allograft. Moderate synovitis and scar tissue were debrided from both the peroneus longus and brevis. At her 6-month postoperative clinic visit, the patient returned to full activity with a pain scale of $1 / 10$. On physical exam, she demonstrated full ROM without laxity. She had $5 / 5$ peroneal strength for the first time since her initial injury.

\section{TECHNIQUE}

The patient is placed supine on the operating table in a supine or sloppy lateral position. A longitudinal incision is centered over the course of the peroneal tendons, beginning $1 \mathrm{~cm}$ posterior and $4 \mathrm{~cm}$ proximal to the tip of the fibula. It is extended to the base of the fifth metatarsal. Carefully identify and protect the sural nerve in the distal aspect of the incision. The surgeon carefully dissects through the extensive scar tissue.

If present, the peroneal sheath is identified. The sheath is incised proximally through the superior peroneal retinaculum to the musculotendinous junction. Distally, the sheath is opened as far as needed. The proximal

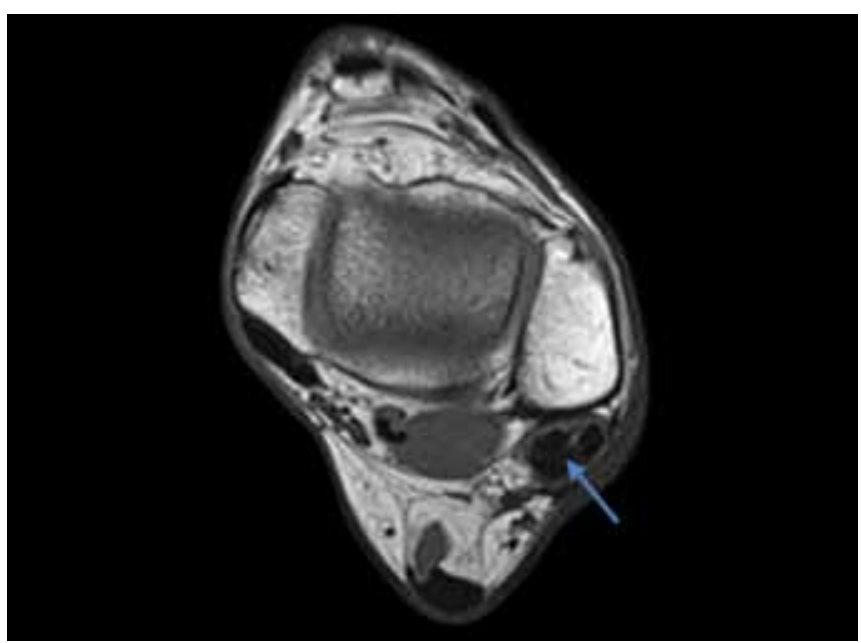

Fig. 2: T1 MRI revealing acute tear in peroneus brevis (arrow) 
peroneus muscle is identified and freed from the surrounding tissue. All diseased distal peroneal tendon tissue is debrided until healthy-appearing tendon is encountered. The distal tendon stump is identified (Fig. 3).

Next, the defect length is measured and the appropriately-sized peroneal tendon or semitendinosus allograft is thawed. One should make sure not to use an allograft length longer than the defect size.

The foot is placed in neutral inversion and eversion as well as neutral ankle dorsiflexion to approximate the appropriate muscle-tendon unit tension. The proximal muscle is pulled distally, noting the length equivalent to $50 \%$ of its excursion. Using a Pulvertaft weave, the allograft is secured to the proximal muscle and tendon using a braided nonabsorbable suture (Fig. 4).

Distally, again identify the stump of the peroneal brevis tendon. The allograft length is set from the remaining gap length when the proximal muscle and tendon are held in this position. If there is an adequate stump distally, the allograft is secured using a Pulvertaft weave and a braided nonabsorbable suture (Fig. 5). If there is an avulsion of the peroneus brevis tendon from the 5th metatarsal, then a bleeding bone bed is made at the anatomic footprint of the base of the fifth metatarsal. A $3.5 \mathrm{~mm}$ suture anchor is then used to secure the allograft to the bone.

The peroneal tendon sheath is closed with absorbable braided sutures and the remainder of the incision is closed in the usual fashion. A sterile dressing and bulky Jones splint is applied.

\section{Alternative to Allograft}

In situations where an allograft tendon is not available, a gracilis or semitendinosis autograft from the ipsilateral side of the leg can be harvested. The implantation and the use of the autograft would be as indicated above.

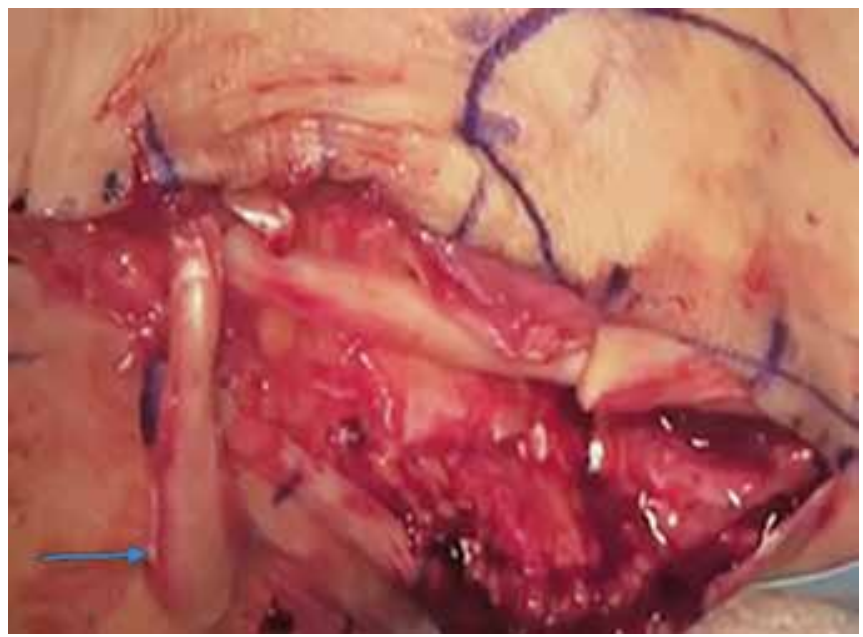

Fig. 3: Diseased peroneal tendon excised, leaving proximal and distal (arrow) stumps for allograft attachment

\section{Postoperative Protocol}

Sutures are removed at 2 weeks, and a nonweight-bearing short-leg cast is applied. At 4 weeks, weight-bearing is progressed to as tolerated in a controlled ankle motion boot. Patients are allowed to remove the boot to dorsiflex and plantar flex the ankle. At 6 weeks, the ankle is protected in an AirCast stirrup (DJO, Vista, CA) for ambulation. Active inversion and eversion (against no resistance) are permitted after 6 weeks. At 12 weeks, a physical therapy strengthening program is prescribed. Unprotected shoe wear is permitted.

\section{DISCUSSION}

Allograft reconstruction has been recently shown to be an effective treatment for chronic peroneal tendinopathy. ${ }^{19}$ Data on 14 patients with allograft reconstruction with a mean follow-up of 17 months was reviewed. All wounds healed without complication. All patients had improved eversion strength postoperatively, and $64 \%$ of patients achieved $5 / 5$ eversion strength. There were no signs or symptoms of lateral ankle instability and importantly, all patients returned to preoperative activity levels. Mean postoperative lower extremity functional and SF-12 scores significantly increased, whereas the average VAS score decreased.

Many techniques are available for peroneal reconstruction including: a two-stage reconstruction using a Hunter rod and flexor hallucis longus (FHL) transfer, ${ }^{20}$ a single-stage reconstruction using a FHL or FDL transfer, ${ }^{21}$ and an acellular dermal matrix augmentation of tears. ${ }^{22}$

In the two-stage reconstruction, the diseased peroneals are excised and a $6 \mathrm{~mm}$ silicon rod is placed into the bed of the peroneal sheath. The Hunter's rod is secured to the remaining stump of the peroneus brevis tendon. Roughly 3 months later, the FHL tendon is harvested.

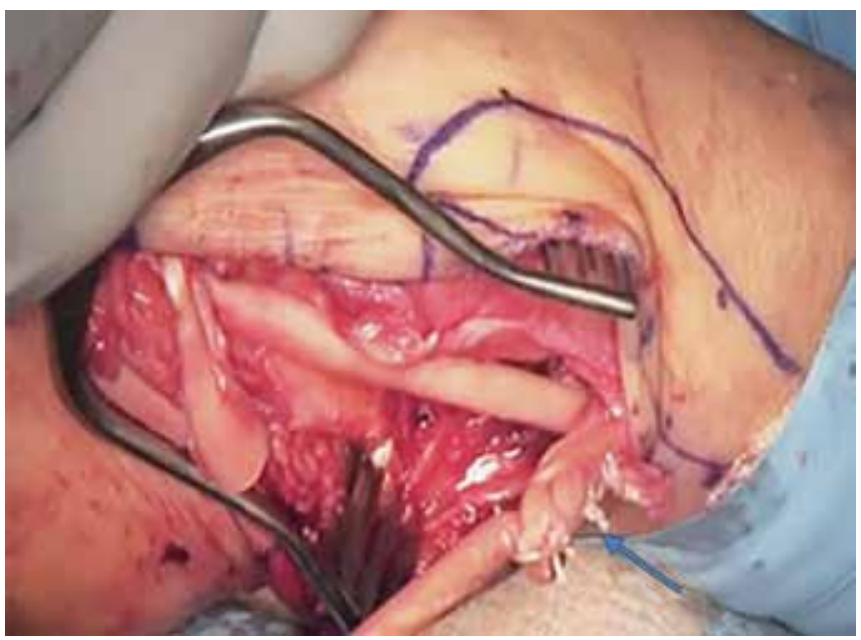

Fig. 4: The allograft is secured using Pulvertaft weave to proximal peroneal brevis tendon (arrow) 


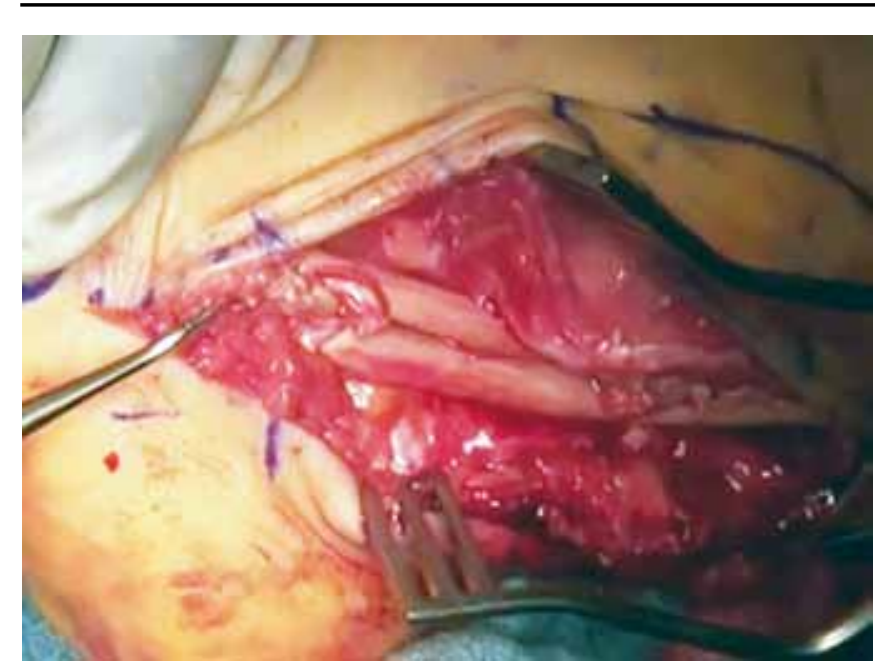

Fig. 5: The allograft secured both proximally and distally to length

The proximal portion of the rod is identified and then FHL is attached to it. The Hunter's rod is detached from the distal site and is pulled distally, allowing the FHL tendon to slide into the newly formed tendon sheath. The FHL is then secured to the stump. Seven patients at 8-year follow-up were reviewed. ${ }^{20}$ Six patients reported a complete relief of preoperative symptoms and a return to preinjury levels of activity. One worker's compensation patient had continued pain and ambulated with a molded ankle-foot orthosis. Overall, there were five excellent results, one good result and one fair result. In this series, the peroneus longus was not addressed since the authors felt that it was too scarred down and that plantar dissection would cause undue harm. They did not believe the loss of the dynamic peroneus longus function contributed to the functional outcomes.

The FHL reconstruction technique does not restore function of both peroneal tendons, since the distal portion of the peroneus longus tendon is too enmeshed in scar to serve as a viable insertion point for the FHL tendon. The intercalary allograft reconstruction technique seeks to restore the muscle-tendon unit without sacrificing normal muscles/tendons that may adversely affect gait mechanics. Using allografts for the reconstruction decreases operative time, eliminates donor site morbidity and gives the surgeon a greater variety of graft sizes. In addition, we have found that these patients have a better normalization of their eversion strength.

Eight patients were treated with a single-stage flexor tendon transfer for nonreconstructable peroneal tendon tears. ${ }^{21}$ In this procedure, once the peroneal tendons were debrided, the FHL or FDL was harvested in the Knot of Henry. The muscle belly and tendon were freed proximally before the tendon was attached to the base of the 5 th metatarsal through a vertical bone tunnel. The tendon is secured with the flexor in maximal tension and the foot in maximal eversion. At final follow-up, mean AOFAS hindfoot scores significantly increased while mean VAS scores significantly decreased. One patient developed a transient peroneal nerve palsy, and one patient underwent a subsequent calcaneal osteotomy. Eighty-eight percent of patients returned to preoperative activity levels, and no patient required daily bracing. These same patients were satisfied with their outcome.

In a study looking at acellular dermal matrix augmentation, four of eleven patients had a procedure to use the graft to bridge the gap between the normal portions of the tendon. The remainder of the patients had an isolated tear of the brevis or longus. In these patients, the graft was used as an augment as it was sutured around the repair. Postoperatively, eversion strength was within one half grade when compared to the contralateral side in all patients (-5/5 in 5 patients and $5 / 5$ in 6 ). All patients were able to perform a single-heel rise and walk on their tiptoes. All patients were satisfied with their outcomes and returned to their normal levels of sport and 10 of the 11 patients had complete resolution of pain.

\section{CONCLUSION}

Chronic lateral ankle pain necessitates the evaluation of the peroneal tendons. Underlying pathology can be variable, and the nonsurgical and surgical options for the patient must be tailored to the presentation. Surgical options vary from primary repair, tenodesis, allograft or autograft reconstruction and tendon transfer depending on the patient findings.

\section{REFERENCES}

1. Bassett FH, 3rd, Speer KP. Longitudinal rupture of the peroneal tendons. Am J Sports Med 1993 May-Jun;21(3):354-357.

2. Brage ME, Hansen ST, Jr. Traumatic subluxation/dislocation of the peroneal tendons. Foot Ankle 1992 Sep;13(7):423-431.

3. Dombek MF, Lamm BM, Saltrick K, Mendicino RW, Catanzariti AR. Peroneal tendon tears: a retrospective review. J Foot Ankle Surg 2003 Sep-Oct;42(5):250-258.

4. Burman M. Stenosing tendovaginitis of the foot and ankle; studies with special reference to the stenosing tendovaginitis of the peroneal tendons of the peroneal tubercle. AMA Arch Surg 1953 Nov;67(5):686-698.

5. Cox D, Paterson FW. Acute calcific tendinitis of peroneus longus. J Bone Joint Surg Br 1991 Mar;73(2):342.

6. Demetracopoulos CA, Vineyard JC, Kiesau CD, Nunley JA, 2nd. Long-term results of debridement and primary repair of peroneal tendon tears. Foot Ankle Int 2014 Mar;35(3):252-257.

7. Sobel M, Geppert MJ, Hannafin JA, Bohne WH, Arnoczky SP. Microvascular anatomy of the peroneal tendons. Foot Ankle 1992 Oct;13(8):469-472.

8. Sammarco GJ, DiRaimondo CV. Chronic peroneus brevis tendon lesions. Foot Ankle 1989 Feb;9(4):163-170.

9. Edwards M. The relations of the peroneal tendons to the fibula, calcaneus, and cuboideum. Am J Anat 1928;42:213-253.

10. Lamm BM, Myers DT, Dombek M, Mendicino RW, Catanzariti AR, Saltrick K. Magnetic resonance imaging and surgical correlation of peroneus brevis tears. J Foot Ankle Surg 2004 Jan-Feb;43(1):30-36. 
11. Sobel M, Geppert MJ, Olson EJ, Bohne WH, Arnoczky SP. The dynamics of peroneus brevis tendon splits: a proposed mechanism, technique of diagnosis, and classification of injury. Foot Ankle 1992 Sep;13(7):413-422.

12. Beck E. Operative treatment of recurrent dislocation of the peroneal tendons. Arch Orthop Trauma Surg 1981;98(4): 247-250.

13. Kojima Y, Kataoka Y, Suzuki S, Akagi M. Dislocation of the peroneal tendons in neonates and infants. Clin Orthop Relat Res 1991 May;266:180-184.

14. Gray JM, Alpar EK. Peroneal tenosynovitis following ankle sprains. Injury $2001 \mathrm{Jul} ; 32(6): 487-489$.

15. Hyer CF, Dawson JM, Philbin TM, Berlet GC, Lee TH. The peroneal tubercle: description, classification, and relevance to peroneus longus tendon pathology. Foot Ankle Int 2005 Nov;26(11):947-950.

16. Sobel M, Levy ME, Bohne WH. Congenital variations of the peroneus quartus muscle: an anatomic study. Foot Ankle 1990 Oct;11(2):81-89.
17. Zammit J, Singh D. The peroneus quartus muscle. Anatomy and clinical relevance. J Bone Joint Surg Br 2003 Nov;85(8): 1134-1137.

18. Redfern D, Myerson M. The management of concomitant tears of the peroneus longus and brevis tendons. Foot Ankle Int 2004 Oct;25(10):695-707.

19. Mook WR, Parekh SG, Nunley JA. Allograft reconstruction of peroneal tendons: operative technique and clinical outcomes. Foot Ankle Int 2013 Sep;34(9):1212-1220.

20. Wapner KL, Taras JS, Lin SS, Chao W. Staged reconstruction for chronic rupture of both peroneal tendons using Hunter rod and flexor hallucis longus tendon transfer: a long-term follow-up study. Foot Ankle Int 2006 Aug;27(8):591-597.

21. Jockel JR, Brodsky JW. Single-stage flexor tendon transfer for the treatment of severe concomitant peroneus longus and brevis tendon tears. Foot Ankle Int 2013 May;34(5):666-672.

22. Rapley JH, Crates J, Barber A. Mid-substance peroneal tendon defects augmented with an acellular dermal matrix allograft. Foot Ankle Int 2010 Feb;31(2):136-140. 